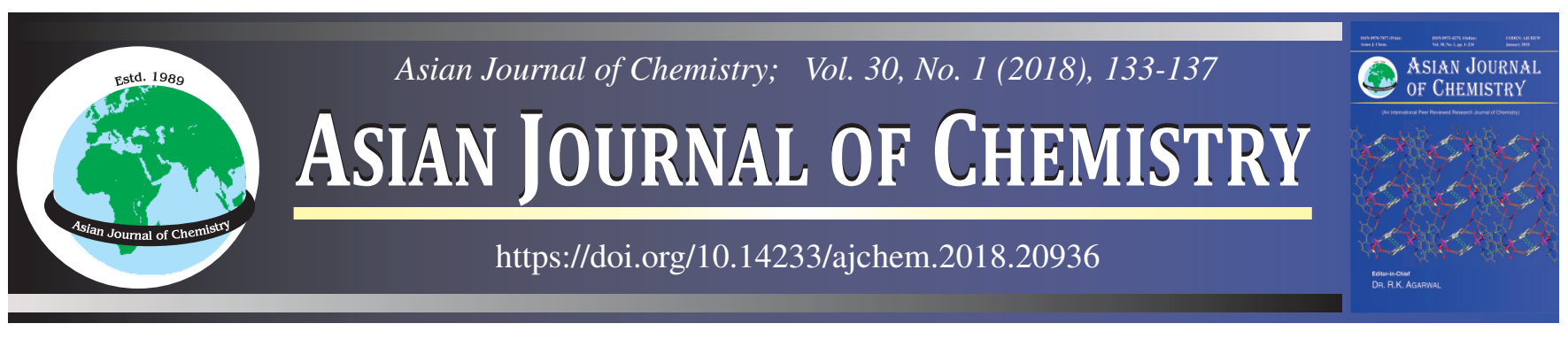

\title{
Microwave Synthesis and Antibacterial Activities of New Imidazolidines Derived from 2-Aminobenzothiazole
}

\author{
Zeid Hassan Abood*, Hussein Ali Qabel, Hayder Raheem Ali and Osama Hameed Rasheed
}

Chemistry Department, College of Science, University of Kerbala, Kerbala, Iraq

*Corresponding author: E-mail: zeid.ab2013@yahoo.com

\begin{abstract}
2-Aminobenzothiazole (1) was converted to the corresponding diazonium salt which was introduced in coupling reaction with alkaline solution of 2-hydroxybenzaldehyde as coupling reagent to give azo-benzothiazole derivative (2) bearing aldehyde group. The resulting aldehyde (2) was introduced in condensation reactions with the primary aromatic amines including (4-nitroaniline, 3-nitroaniline, 4-hydroxyaniline, 4-methoxyaniline, 2-methoxyaniline, 4-bromoaniline, 4-chloroaniline and 2,4-dichloroaniline) using microwave irradiation technique in absolute ethanol to produce eight imine derivatives of benzothiazole (3a-h), respectively. Treatment of the resulting imines (3a-h) with glycine using microwave irradiation in tetrahydrofuran afforded eight new imidazolidines (4a-h) substituted with benzothiazole moiety, respectively. Preliminary in vitro antibacterial activity of the target compounds were investigated using two types of bacteria, Staphylococcus aureus (Gram-positive) and Escherichia coli (Gram-negative). The results indicated that the newly synthesized imidazolidines (compounds $\mathbf{4 a}, \mathbf{4 b}, \mathbf{4 c}, \mathbf{4 d}, \mathbf{4 e}, \mathbf{4 g}$ and $\mathbf{4 h}$ ) exhibited greater activities than gentamycin against Gram-positive bacteria. On the other hand, compounds $\mathbf{4 d}$ and $\mathbf{4 h}$ were also showed better activities against Gram-negative bacteria when compared with that of the control drug (gentamycin).
\end{abstract}

Keywords: Imidazolidines, Imines, Benzothiazoles, Azo, Antibacterial activity.

Keywords: Imidazolidines, Imines, Benzothiazoles, Azo, Antibacterial activity.

\section{INTRODUCTION}

Imidazolidines (saturated imidazoles), also known as tetrahydroimidazoles are biologically active nitrogen containing heterocyclic moiety which have been reported to shown wide array of significant bioactivities [1]. Moreover, the construction of optically active stereogenic center in a saturated heterocycle such as imidazolidine [2]. Imidazolidines have found therapeutic applications in drugs such as the well established phenytoin [3]. Among the wide range of biological activities of substituted hydantoins we can cite antidiabetic, anti-inflammatory, antiviral, GHS and CB1 receptor antagonists ${ }^{3}$ and more. Imidazolidine ring of such compounds yields molecules with variety of pharmacological properties, including anti-inflammatory, antifungal, antibacterial, hypoglycemic, schistosomicides anticancer activities [4], antihypertensive/anaesthetic [5], antiarrhythmic [6], modulation of anxiety [7], antinociceptive activity [8], anti-Alzheimer's [9], antimicrobial [10], antinociceptive [11], antitumor [12], anti-trypanosoma cruzi [13], anti-depressant [14] and antifungal drugs [15]. Benzothiazole consists of thiazole ring fused with benzene and is also called 1-thia-3-azaindene. It is a weak base and thermally stable molecule [16]. In the 1990s, various pharmacological investi- gations of newly synthesized benzothiazoles demonstrated interesting pharmacological activities and led to the development of new medications for treating diseases. They were studied extensively for their antiallergic, antiinflammatory [17], analgesic [18], antitumor [19], antiproliferative activities [20]. Benzothiazoles have been reported to be antioxidant agents [21], antitubercular [22], antipsychotic [23], anticonvulsant [24], anticancer agents [25], antiparasite activity [26], antimalaria [27], etc.

\section{EXPERIMENTAL}

The chemicals were used as provided from Fluka, Sigma Aldrich and Merck. Microwave reactions were performed on domestic microwave oven in crucible. Analytical TLC was performed with silica gel $60 \mathrm{~F}_{254}$ plates. The reactions were monitored by TLC and visualized by development of the TLC plates with iodine vapour. Melting points were recorded on an Electro thermal Stuart SMP 30 capillary melting point apparatus and are uncorrected. Infrared spectra were recorded on SHIMADZU FTIR-8400S infrared spectrophotometer as potassium bromide discs. ${ }^{1} \mathrm{H}$ NMR spectra was collected on Avance III 500, NMR spectrometer, Bruker, Germany at $500 \mathrm{MHz}$ in DMSO- $d_{6}$ as 
solvent and TMS as an internal standard at a Faculty of Science, University of Tarbiat Modares, Iran. (CHNS) Elemental analysis was carried out with Perkin Elmer 300A elemental analyzer at a Faculty of Science, University of Tarbiat Modares, Iran.

(E)-5-(Benzo[d]thiazol-2-yldiazenyl)-2-hydroxy benzaldehyde (2) was synthesized following the method described by Acton [28] as dark brown solid, m.p. $141-143{ }^{\circ} \mathrm{C}$, yield 60 $\%$; IR ( $\left.\mathrm{cm}^{-1}\right): 3429_{\text {br }} \mathrm{v}(\mathrm{O}-\mathrm{H}), 3068 \mathrm{v}(\mathrm{C}-\mathrm{H})$ benzene, 2856 and $2715 v(\mathrm{C}-\mathrm{H})$ aldehyde, $1645 \mathrm{v}(\mathrm{C}=\mathrm{O})$ aldehyde, $1610 \mathrm{v}(\mathrm{C}=\mathrm{N})$ benzothiazole, 1502 and $1475 \mathrm{v}(\mathrm{C}=\mathrm{C})$ benzene, $1375 \mathrm{v}(\mathrm{N}=\mathrm{N})$, 759 \&(o.o.p.C-H) benzene.

General procedure for synthesis of imines (3a-h): All reactions were carried out on domestic microwave oven in crucible. Reactions contained the azoaldehyde derivative (2) $(0.283 \mathrm{~g}, 1 \mathrm{mmol})$, equimolar amount $(1 \mathrm{mmol})$ of aniline derivatives (4-nitroaniline, 3-nitroaniline, 4-hydroxyaniline, 4-methoxyaniline, 2-methoxyaniline, 4-bromoaniline, 4chloroaniline and 2,4-dichloroaniline respectively) and absolute ethanol ( $1 \mathrm{~mL})$. The crucible was introduced to the center of a domestic microwave oven and then heated (300-350 W) for 25-30 min. TLC ( $n$-hexane: EtOAc) showed that the reactions were completed. The products were washed with diethyl ether and recrystallized from ethanol.

4-[(E)-Benzo[d]thiazol-2-yldiazenyl]-2-[(E)-)((4-nitrophenyl)imino)methyl]phenol (3a): IR $\left(\mathrm{cm}^{-1}\right)$ : $3371 \mathrm{v}(\mathrm{O}-\mathrm{H})$, $3064 v(\mathrm{C}-\mathrm{H})$ benzene, $1608 \mathrm{v}(\mathrm{C}=\mathrm{N})$ imine, $1589 \mathrm{v}(\mathrm{C}=\mathrm{N})$ benzothiazole, $1514 \operatorname{vas}\left(\mathrm{NO}_{2}\right), 1448 \mathrm{v}(\mathrm{C}=\mathrm{C})$ benzene, 1404

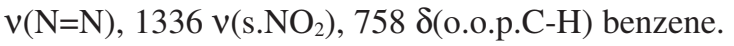

4-[(E)-Benzo[d]thiazol-2-yldiazenyl]-2-[(E)-((3-nitrophenyl)imino)methyl]phenol (3b): IR $\left(\mathrm{cm}^{-1}\right)$ : $3246 \mathrm{v}(\mathrm{O}-\mathrm{H})$, $3088 v(\mathrm{C}-\mathrm{H})$ benzene, $1604 \mathrm{v}(\mathrm{C}=\mathrm{N})$ imine and $\mathrm{v}(\mathrm{C}=\mathrm{N})$ benzothiazole, vib. coupling, 1573 and 1502 and $1444 \mathrm{v}(\mathrm{C}=\mathrm{C})$ benzene, 1525 vas $\left(\mathrm{NO}_{2}\right), 1396 \mathrm{v}(\mathrm{N}=\mathrm{N}), 1354 \mathrm{v}\left(\mathrm{s} . \mathrm{NO}_{2}\right), 758$ $\delta$ (o.o.p.C-H) benzene.

4-[(E)-Benzo[d] thiazol-2-yldiazenyl]-2-[(E)-((4-hydroxyphenyl)imino)methyl]phenol (3c): IR $\left(\mathrm{cm}^{-1}\right): 3369$ and 3298 $\mathrm{v}(\mathrm{O}-\mathrm{H}), 3032 \mathrm{v}(\mathrm{C}-\mathrm{H})$ benzene, $1610 \mathrm{v}(\mathrm{C}=\mathrm{N}$, imine and $v(\mathrm{C}=\mathrm{N})$ benzothiazole, vib. coupling $), 1514$ and $1460 v(\mathrm{C}=\mathrm{C})$ benzene, $1379 v(\mathrm{~N}=\mathrm{N}), 827 \delta$ (o.o.p.C-H) benzene.

4-[(E)-Benzo[d]thiazol-2-yldiazenyl]-2-[(E)-((4-methoxyphenyl)imino)methyl]phenol (3d): IR $\left(\mathrm{cm}^{-1}\right): 3371$ and 3298 $v(\mathrm{O}-\mathrm{H}), 3037 \mathrm{v}(\mathrm{C}-\mathrm{H})$ benzene, $2949 \mathrm{vas}\left(\mathrm{CH}_{3}\right), 1622 \mathrm{v}(\mathrm{C}=\mathrm{N})$ imine, $1595 \mathrm{v}(\mathrm{C}=\mathrm{N})$ benzothiazole, 1512 and $1456 \mathrm{v}(\mathrm{C}=\mathrm{C})$ benzene, $1392 v(\mathrm{~N}=\mathrm{N}), 821 \delta($ o.o.p.C-H) benzene.

4-[(E)-Benzo[d]thiazol-2-yldiazenyl]-2-[(E)-((2-methoxyphenyl)imino)methyl]phenol (3e): IR $\left(\mathrm{cm}^{-1}\right)$ : 3462 and 3265 $\mathrm{v}(\mathrm{O}-\mathrm{H}), 3064 \mathrm{v}(\mathrm{C}-\mathrm{H})$ benzene, $2968 \mathrm{vas}\left(\mathrm{CH}_{3}\right), 2870 \mathrm{v}\left(\mathrm{s} . \mathrm{CH}_{3}\right)$, $1633 v(\mathrm{C}=\mathrm{N})$ imine, $1608 v(\mathrm{C}=\mathrm{N})$ benzothiazole, 1533, 1500 and $1444 \mathrm{v}(\mathrm{C}=\mathrm{C})$ benzene, $1363 \mathrm{v}(\mathrm{N}=\mathrm{N}), 831 \delta$ (o.o.p.C-H) benzene.

4-[(E)-Benzo[d]thiazol-2-yldiazenyl]-2-[(E)-((4-bromophenyl)imino)methyl]phenol (3f): IR $\left(\mathrm{cm}^{-1}\right)$ : 3369 and 3261 $\mathrm{v}(\mathrm{O}-\mathrm{H}), 3066 \mathrm{v}(\mathrm{C}-\mathrm{H})$ benzene, $1633 \mathrm{v}(\mathrm{C}=\mathrm{N})$ imine, 1608 $v(\mathrm{C}=\mathrm{N})$ benzothiazole, 1506 and $1489 \mathrm{v}(\mathrm{C}=\mathrm{C})$ benzene, 1363 $v(\mathrm{~N}=\mathrm{N}), 821 \delta$ (o.o.p.C-H) benzene.

4-[(E)-Benzo[d]thiazol-2-yldiazenyl]-2-[(E)-((4-chlorophenyl)imino)methyl]phenol (3g): IR $\left(\mathrm{cm}^{-1}\right)$ : 3369 and 3263 $\mathrm{v}(\mathrm{O}-\mathrm{H}), 3064 \mathrm{v}(\mathrm{C}-\mathrm{H})$ benzene, $1633 \mathrm{v}(\mathrm{C}=\mathrm{N})$ imine, 1606
$v(\mathrm{C}=\mathrm{N})$ benzothiazole, 1537,1496 and $1442 v(\mathrm{C}=\mathrm{C})$ benzene, $1367 v(\mathrm{~N}=\mathrm{N}), 821 \delta$ (o.o.p.C-H) benzene.

4-[(E)-Benzo[d]thiazol-2-yldiazenyl $]-2-[(E)-((2,4-$ dichlorophenyl)imino)methyl]phenol (3h): IR $\left(\mathrm{cm}^{-1}\right): 3470$ and $3265 v(\mathrm{O}-\mathrm{H}), 3061 \mathrm{v}(\mathrm{C}-\mathrm{H})$ benzene, $1633 \mathrm{v}(\mathrm{C}=\mathrm{N})$ imine, $1608 v(\mathrm{C}=\mathrm{N})$ benzothiazole, 1570, 1533, 1500 and 1444 $v(\mathrm{C}=\mathrm{C})$ benzene, $1363 v(\mathrm{~N}=\mathrm{N}), 831 \delta$ (o.o.p.C-H) benzene.

General procedure for synthesis of imidazolidines (4ah): A mixture of equimolar amounts of imine derivatives (3a-h) (1 mmol) and glycine $(0.075 \mathrm{~g}, 1 \mathrm{mmol})$ in tetrahydrofuran $(1 \mathrm{~mL})$ was heated $(550-610 \mathrm{~W})$ in microwave oven for $20-25$ min. TLC ( $n$-hexane:EtOAc) showed that the reactions were completed. The products were washed with diethyl ether and recrystallized from ethanol.

(E)-2-[5-(Benzo[d]thiazol-2-yldiazenyl)-2-hydroxyphenyl]-3-(4-nitrophenyl)imidazolidin-4-one (4a): IR $\left(\mathrm{cm}^{-1}\right)$ : $3365_{\text {br }} v(\mathrm{O}-\mathrm{H})$ and $\mathrm{v}(\mathrm{N}-\mathrm{H})$ imidazolidine, vib. coupling $), 3074$ $\mathrm{v}(\mathrm{C}-\mathrm{H})$ benzene, $1708 \mathrm{v}(\mathrm{C}=\mathrm{O})$ imidazolidine, $1680 \delta(\mathrm{N}-\mathrm{H})$ imidazolidine, 1539 and $1456 \mathrm{v}(\mathrm{C}=\mathrm{C})$ benzene, $1516 \mathrm{v}_{\mathrm{as}}\left(\mathrm{NO}_{2}\right)$, $1419 v(\mathrm{~N}=\mathrm{N}), 1321 \mathrm{v}_{\mathrm{s}}\left(\mathrm{NO}_{2}\right), 756 \delta$ (o.o.p.C-H) benzene; ${ }^{1} \mathrm{H}$ NMR: $\delta(\mathrm{ppm})=3.69\left(\mathrm{~d}, J=6.5 \mathrm{~Hz}, 2 \mathrm{H}, \mathrm{CH}_{2}\right)$ imidazolidine, $6.63(\mathrm{~d}, J=8.1 \mathrm{~Hz}, 1 \mathrm{H}, \mathrm{CH})$ imidazolidine, 7.37-7.96 $(11 \mathrm{H}$, Ar-H), 8.14 (s, 1H, N-H) imidazolidine, 9.48 (s, 1H, O-H). The singlet signals around $2.49 \mathrm{ppm}$ and $3.44 \mathrm{ppm}$ attributed to DMSO and absorbed $\mathrm{H}_{2} \mathrm{O}$ in DMSO, respectively; Anal. calcd. for $\mathrm{C}_{22} \mathrm{H}_{16} \mathrm{~N}_{6} \mathrm{O}_{4} \mathrm{~S}$ : C, 57.39; H, 3.50; N, 18.25; S, 6.96; Found C, 56.99; H, 3.45; N, 18.02; S, 6.96.

(E)-2-[5-(Benzo[ $d]$ thiazol-2-yldiazenyl)-2-hydroxyphenyl]-3-(3-nitrophenyl)imidazolidin-4-one (4b): IR $\left(\mathrm{cm}^{-1}\right)$ : $3402_{\text {br }} v(\mathrm{O}-\mathrm{H})$ and $\mathrm{v}(\mathrm{N}-\mathrm{H})$ imidazolidine, vib. coupling $), 3074$ $v(\mathrm{C}-\mathrm{H})$ benzene, $1710 \mathrm{v}(\mathrm{C}=\mathrm{O})$ imidazolidine, $1683 \delta(\mathrm{N}-\mathrm{H})$ imidazolidine, $1604 \mathrm{v}(\mathrm{C}=\mathrm{N})$ benzothiazole, $1508 \mathrm{v}_{\mathrm{as}}\left(\mathrm{NO}_{2}\right)$, 1456 and $1421 \mathrm{v}(\mathrm{C}=\mathrm{C})$ benzene, $1384 \mathrm{v}(\mathrm{N}=\mathrm{N}), 1323 \mathrm{v}_{\mathrm{s}}\left(\mathrm{NO}_{2}\right)$, $759 \delta$ (o.o.p.C-H) benzene; ${ }^{1} \mathrm{H}$ NMR: $\delta(\mathrm{ppm})=3.69(\mathrm{~d}, J=$ $\left.20.0 \mathrm{~Hz}, 2 \mathrm{H}, \mathrm{CH}_{2}\right)$ imidazolidine, $6.62(\mathrm{~s}, 1 \mathrm{H}, \mathrm{CH})$ imidazolidine, 6.92-7.97 (11H, Ar-H), 8.12 (s, 1H, N-H) imidazolidine, $9.56(\mathrm{~s}, 1 \mathrm{H}, \mathrm{O}-\mathrm{H})$. The singlet signals around $2.49 \mathrm{ppm}$ and $3.42 \mathrm{ppm}$ assigned to DMSO and absorbed $\mathrm{H}_{2} \mathrm{O}$ in DMSO, respectively; Anal. calcd. for $\mathrm{C}_{22} \mathrm{H}_{16} \mathrm{~N}_{6} \mathrm{O}_{4} \mathrm{~S}: \mathrm{C}, 57.39 ; \mathrm{H}, 3.50$; N, 18.25; S, 6.96; Found C, 57.01; H, 3.45; N, 18.12; S, 6.77.

(E)-2-[5-(Benzo[d]thiazol-2-yldiazenyl)-2-hydroxyphenyl]-3-(4-hydroxyphenyl)imidazolidin-4-one (4c): IR $\left(\mathrm{cm}^{-1}\right): 3200_{\mathrm{br}} \mathrm{v}(\mathrm{O}-\mathrm{H})$ and $v(\mathrm{~N}-\mathrm{H})$ imidazolidine, vib. coupling, $3080 v(\mathrm{C}-\mathrm{H})$ benzene, $1670 v(\mathrm{C}=\mathrm{O})$ and $\delta(\mathrm{N}-\mathrm{H})$ imidazolidine, vib. coupling, $1616 \mathrm{v}(\mathrm{C}=\mathrm{N})$ benzothiazole, 1539, 1514 and $1454 v(\mathrm{C}=\mathrm{C})$ benzene, $1417 v(\mathrm{~N}=\mathrm{N}), 893 \delta$ (o.o.p.C-H) benzene; ${ }^{1} \mathrm{H}$ NMR: $\delta(\mathrm{ppm})=3.69\left(\mathrm{~d}, J=11.3 \mathrm{~Hz}, 2 \mathrm{H}, \mathrm{CH}_{2}\right)$ imidazolidine, $6.63(\mathrm{~d}, J=7.3 \mathrm{~Hz}, 1 \mathrm{H}, \mathrm{CH})$ imidazolidine, 6.89-7.99 (11H, Ar-H), 8.12 (s, 1H, N-H) imidazolidine, 9.53 $(\mathrm{s}, 2 \mathrm{H}, 2 \times \mathrm{O}-\mathrm{H})$. The singlet signals around $2.49 \mathrm{ppm}$ and 3.43 ppm due to DMSO and absorbed $\mathrm{H}_{2} \mathrm{O}$ in DMSO, respectively; Anal. calcd. for $\mathrm{C}_{22} \mathrm{H}_{17} \mathrm{~N}_{5} \mathrm{O}_{3} \mathrm{~S}$ : C, 61.24; H, 3.97; N, 16.23; S, 7.43; Found C, 60.88; H, 3.61; N, 16.30; S, 7.33.

(E)-2-[5-(Benzo[d]thiazol-2-yldiazenyl)-2-hydroxyphenyl]-3-(4-methoxyphenyl)imidazolidin-4-one (4d): IR $\left(\mathrm{cm}^{-1}\right): 3250_{\mathrm{br}} \mathrm{v}(\mathrm{O}-\mathrm{H})$ and $\mathrm{v}(\mathrm{N}-\mathrm{H})$ imidazolidine, vib. coupling, $3074 v(\mathrm{C}-\mathrm{H})$ benzene, $2964 v_{\text {as }}\left(\mathrm{CH}_{3}\right) 1670 v(\mathrm{C}=\mathrm{O})$ and $\delta(\mathrm{N}-\mathrm{H})$ imidazolidine, vib. coupling, $1602 \mathrm{v}(\mathrm{C}=\mathrm{N})$ benzothiazole, 
1514 and $1458 v(\mathrm{C}=\mathrm{C})$ benzene, $1421 \mathrm{v}(\mathrm{N}=\mathrm{N}), 829 \delta$ (o.o.p.C$\mathrm{H})$ benzene; ${ }^{1} \mathrm{H}$ NMR: $\delta(\mathrm{ppm})=3.69(\mathrm{~d}, J=12.8 \mathrm{~Hz}, 2 \mathrm{H}$, $\mathrm{CH}_{2}$ ) imidazolidine, $3.94\left(\mathrm{~s}, 3 \mathrm{H}, \mathrm{O}-\mathrm{CH}_{3}\right), 6.63(\mathrm{~d}, J=8.3 \mathrm{~Hz}$, $1 \mathrm{H}, \mathrm{CH})$ imidazolidine, 6.79-7.98 (11H, Ar-H), 8.15 (s, 1H, $\mathrm{N}-\mathrm{H})$ imidazolidine, $9.52(\mathrm{~s}, 1 \mathrm{H}, \mathrm{O}-\mathrm{H})$. The singlet signals around 2.49 and $3.42 \mathrm{ppm}$ assigned to DMSO and absorbed $\mathrm{H}_{2} \mathrm{O}$ in DMSO, respectively; Anal. calcd. for $\mathrm{C}_{23} \mathrm{H}_{19} \mathrm{~N}_{5} \mathrm{O}_{3} \mathrm{~S}: \mathrm{C}$, 62.01; H, 4.30; N, 15.72; S, 7.20; Found C, 61.88; H, 4.26; N, $15.51 ; \mathrm{S}, 7.01$.

(E)-2-[5-(Benzo[d]thiazol-2-yldiazenyl)-2-hydroxyphenyl]-3-(2-methoxyphenyl)imidazolidin-4-one (4e): IR $\left(\mathrm{cm}^{-1}\right): 3192_{\text {br }} v(\mathrm{O}-\mathrm{H})$ and $v(\mathrm{~N}-\mathrm{H})$ imidazolidine, vib. coupling, $3072 \mathrm{v}(\mathrm{C}-\mathrm{H})$ benzene, $1681 \mathrm{v}(\mathrm{C}=\mathrm{O})$ imidazolidine, $1653 \delta(\mathrm{N}-$ $\mathrm{H})$ imidazolidine, $1610 \mathrm{v}(\mathrm{C}=\mathrm{N})$ benzothiazole, 1558, 1541 and $1456 v(\mathrm{C}=\mathrm{C})$ benzene, $1423 \mathrm{v}(\mathrm{N}=\mathrm{N}), 823 \delta$ (o.o.p.C-H) benzene; ${ }^{1} \mathrm{H}$ NMR: $\delta(\mathrm{ppm})=3.69\left(\mathrm{~d}, J=12.8 \mathrm{~Hz}, 2 \mathrm{H}, \mathrm{CH}_{2}\right)$ imidazolidine, 3.83 (s, 3H, O- $\left.\mathrm{CH}_{3}\right), 6.77$ (d, $J=8.3 \mathrm{~Hz}, 1 \mathrm{H}$, $\mathrm{CH})$ imidazolidine, 7.01-7.99 (11H, Ar-H), 8.15 (s, 1H, N$\mathrm{H})$ imidazolidine, $9.62(\mathrm{~s}, 1 \mathrm{H}, \mathrm{O}-\mathrm{H})$. The singlet signals around 2.49 and 3.39 ppm assigned to DMSO and absorbed $\mathrm{H}_{2} \mathrm{O}$ in DMSO, respectively; Anal. calcd. for $\mathrm{C}_{23} \mathrm{H}_{19} \mathrm{~N}_{5} \mathrm{O}_{3} \mathrm{~S}: \mathrm{C}, 62.01$; H, 4.30; N, 15.72; S, 7.20; Found C, 62.37; H, 4.21; N, 15.36; S, 7.11.

(E)-2-[5-(Benzo[d]thiazol-2-yldiazenyl)-2-hydroxyphenyl]-3-(4-bromophenyl)imidazolidin-4-one(4f): IR $\left(\mathrm{cm}^{-1}\right)$ : $3176_{\text {br }} \mathrm{v}(\mathrm{O}-\mathrm{H})$ and $\mathrm{v}(\mathrm{N}-\mathrm{H})$ imidazolidine, vib. coupling, 3063 $v(\mathrm{C}-\mathrm{H})$ benzene, $1666 v(\mathrm{C}=\mathrm{O})$ and $\delta(\mathrm{N}-\mathrm{H})$ imidazolidine, vib. coupling, $1610 v(\mathrm{C}=\mathrm{N})$ benzothiazole, $1508 \mathrm{v}(\mathrm{C}=\mathrm{C})$ benzene, $1415 \mathrm{v}(\mathrm{N}=\mathrm{N}), 823 \delta$ (o.o.p.C-H) benzene; ${ }^{1} \mathrm{H}$ NMR: $\delta(\mathrm{ppm})$ $=3.69\left(\mathrm{~d}, J=23.8 \mathrm{~Hz} 2 \mathrm{H}, \mathrm{CH}_{2}\right)$ imidazolidine, $6.54(\mathrm{~d}, J=$ $8.0 \mathrm{~Hz}, 1 \mathrm{H}, \mathrm{CH})$ imidazolidine, 6.67-8.00 (11H, Ar-H), 8.15 $(\mathrm{s}, 1 \mathrm{H}, \mathrm{N}-\mathrm{H})$ imidazolidine, $9.64(\mathrm{~s}, 1 \mathrm{H}, \mathrm{O}-\mathrm{H})$. The singlet signals around 2.49 and $3.51 \mathrm{ppm}$ due to DMSO and absorbed $\mathrm{H}_{2} \mathrm{O}$ in DMSO, respectively; Anal. calcd. for $\mathrm{C}_{22} \mathrm{H}_{16} \mathrm{~N}_{5} \mathrm{O}_{2} \mathrm{SBr}$ : C, 53.45; H, 3.26; N, 14.17; S, 6.49; Found C, 52.08; H, 3.59; N, 13.93; S, 6.45 .

(E)-2-[5-(Benzo[d]thiazol-2-yldiazenyl)-2-hydroxyphenyl]-3-(4-chlorophenyl)imidazolidin-4-one (4g): IR $\left(\mathrm{cm}^{-1}\right)$ : $3184_{\text {br }} v(\mathrm{O}-\mathrm{H})$ and $v(\mathrm{~N}-\mathrm{H})$ imidazolidine, vib. coupling, 3066 $v(\mathrm{C}-\mathrm{H})$ benzene, $1670 v(\mathrm{C}=\mathrm{O})$ and $\delta(\mathrm{N}-\mathrm{H})$ imidazolidine, vib. coupling, $1610 \vee(\mathrm{C}=\mathrm{N})$ benzothiazole, 1523 and $1508 \mathrm{v}(\mathrm{C}=\mathrm{C})$ benzene, $1417 v(\mathrm{~N}=\mathrm{N}), 893 \delta$ (o.o.p.C-H) benzene; ${ }^{1} \mathrm{H}$ NMR: $\delta(\mathrm{ppm})=3.69\left(\mathrm{~d}, J=23.8 \mathrm{~Hz}, 2 \mathrm{H}, \mathrm{CH}_{2}\right)$ imidazolidine, 6.52 $(\mathrm{d}, J=8.0 \mathrm{~Hz}, 1 \mathrm{H}, \mathrm{CH})$ imidazolidine, 6.67-7.99 $(11 \mathrm{H}, \mathrm{Ar}-$ $\mathrm{H}), 8.15$ (s, 1H, N-H) imidazolidine, 9.17 (s, 1H, O-H). The singlet signals around $2.49 \mathrm{ppm}$ and $3.39 \mathrm{ppm}$ attributed to DMSO and absorbed $\mathrm{H}_{2} \mathrm{O}$ in DMSO, respectively; Anal. calcd. for $\mathrm{C}_{22} \mathrm{H}_{16} \mathrm{~N}_{5} \mathrm{O}_{2} \mathrm{SCl}: \mathrm{C}, 58.73 ; \mathrm{H}, 3.58 ; \mathrm{N}, 15.57$; S, 7.13; Found C, 58.64; H, 3.69; N, 15.23; S, 7.38.

(E)-2-[5-(Benzo[d]thiazol-2-yldiazenyl)-2-hydroxyphenyl]-3-(2,4-dichlorophenyl)imidazolidin-4-one (4h): IR $\left(\mathrm{cm}^{-1}\right): 3232_{\mathrm{br}} \mathrm{v}(\mathrm{O}-\mathrm{H})$ and $\mathrm{v}(\mathrm{N}-\mathrm{H})$ imidazolidine, vib. coupling), $3082 v(\mathrm{C}-\mathrm{H})$ benzene, $1681 v(\mathrm{C}=\mathrm{O})$ imidazolidine, $1653 \delta(\mathrm{N}-$ $\mathrm{H})$ imidazolidine, vib. coupling, $1608 \mathrm{v}(\mathrm{C}=\mathrm{N})$ benzothiazole, 1554, 1541 and $1508 v(\mathrm{C}=\mathrm{C})$ benzene, $1423 v(\mathrm{~N}=\mathrm{N}), 819$ $\delta$ (o.o.p.C-H) benzene; ${ }^{1} \mathrm{H}$ NMR: $\delta(\mathrm{ppm})=3.68(\mathrm{~d}, J=7.4$ $\left.\mathrm{Hz}, 2 \mathrm{H}, \mathrm{CH}_{2}\right)$ imidazolidine, $6.74(\mathrm{~d}, J=8.3 \mathrm{~Hz}, 1 \mathrm{H}, \mathrm{CH})$ imidazolidine, 7.01-8.14 (10H, Ar-H), 9.29 (s, 1H, N-H) imidazolidine, 9.62 (s, 1H, O-H). The singlet signals around 2.49 and 3.35 ppm assigned to DMSO and absorbed $\mathrm{H}_{2} \mathrm{O}$ in DMSO, respectively; Anal. calcd. for $\mathrm{C}_{22} \mathrm{H}_{15} \mathrm{~N}_{5} \mathrm{O}_{2} \mathrm{SCl}_{2}$ : C, 54.55; H, 3.12; N, 14.46; S, 6.62; Found C, 54.21; H, 3.02; N, 14.22; S, 6.31 .

Preliminary antibacterial assay: The antibacterial activities of the newly synthesized imidazolidines (4a-h) were determined by the agar diffusion method [29] using representative Gram (+) and Gram (-) bacteria on tryptic soya agar media. The test microorganisms to evaluate the potential antibacterial activity of the newly synthesized imidazolidines were Staphylococcus aureus (Gram-positive) and Escherichia coli (Gramnegative). The imidazolidines were dissolved in dimethyl sulfoxide to prepare the test solutions of $10 \mathrm{mg} / \mathrm{mL}$ concentration. Gentamycin was used as a reference and the activities were presented as zones of inhibition for each compound.

\section{RESULTS AND DISCUSSION}

Amino group in 2-aminobenzothiazole (1) was diazotized using sodium nitrite and sulfuric acid to generate the corresponding diazonium salt which was directly introduced in coupling reaction with 2-hydroxy benzaldehyde dissolved in sodium hydroxide solution to give azo-benzothiazole derivative (2) containing aldehyde group. Aldehyde group of azo-benzothiazole derivative (2) was condensed with the primary aromatic amines including (4-nitroaniline, 3-nitroaniline, 4-hydroxyaniline, 4-methoxynitroaniline, 2-methoxyaniline, 4-bromoaniline,4-chloroaniline and 2,4-dichloroaniline) using microwave irradiation in absolute ethanol to produce eight imine derivatives of benzothiazole (3a-h) respectively (Schemes I and II). The resulting imines (3a-h) were allowed to react with the glycine a using microwave irradiation leading to the formation of imidazolidine derivatives of benzothiazole (4a-h) in good yields (Table-1).

The chemical structures of the target compounds synthesized were deduced from IR, ${ }^{1} \mathrm{H}$ NMR spectral measurements and (CHNS) elemental analysis and were in good agreement with the proposed structures.

The IR and ${ }^{1} \mathrm{H}$ NMR spectra of the desired compounds $\mathbf{4 a - h}$ were described. The IR spectrum of azo-benzothiazole derivative (2) indicated the absence of a doublet band at 3375 and $3306 \mathrm{~cm}^{-1}$ for $\left(\mathrm{NH}_{2}\right)$ str. in 2-aminobenzothiazole (1) and appearance of the following characteristic bands: the medium band at $1375 \mathrm{~cm}^{-1}$ attributed to azo group $(\mathrm{N}=\mathrm{N})$ str, the broad band at $3429 \mathrm{~cm}^{-1}$ assigned to $(\mathrm{O}-\mathrm{H})$ str, the sharp and strong band at $1645 \mathrm{~cm}^{-1}$ belong to aldehydic $(\mathrm{C}=\mathrm{O})$ str., the benzothiazolic $(\mathrm{C}=\mathrm{N})$ str. appeared as strong band at $1610 \mathrm{~cm}^{-1}$. IR spectra of the benzothiazolicimines (3a-h) showed disappearing the sharp and strong band at $1645 \mathrm{~cm}^{-1}$ for aldehydic $(\mathrm{C}=\mathrm{O})$ str., also disappearing the sharp doublet band for $\left(\mathrm{NH}_{2}\right)$ str. in the starting amines at the general range $3400-3250 \mathrm{~cm}^{-1}$ and appearing absorption band at the range $1633-1604 \mathrm{~cm}^{-1}$ assigned to the iminic $(\mathrm{C}=\mathrm{N})$ str. The benzothiazolic $(\mathrm{C}=\mathrm{N})$ str. appeared at the range $1610-1589 \mathrm{~cm}^{-1}$. The IR spectra of the benzothiazolic-imidazolidines (4a-h) showed the appearance of strong band at the range $1710-1666 \mathrm{~cm}^{-1}$ attributed to the $(\mathrm{C}=\mathrm{O})$ str. of the imidazolidine ring. The spectra also showed the appearance of absorption band at the range $1683-1653 \mathrm{~cm}^{-1}$ 


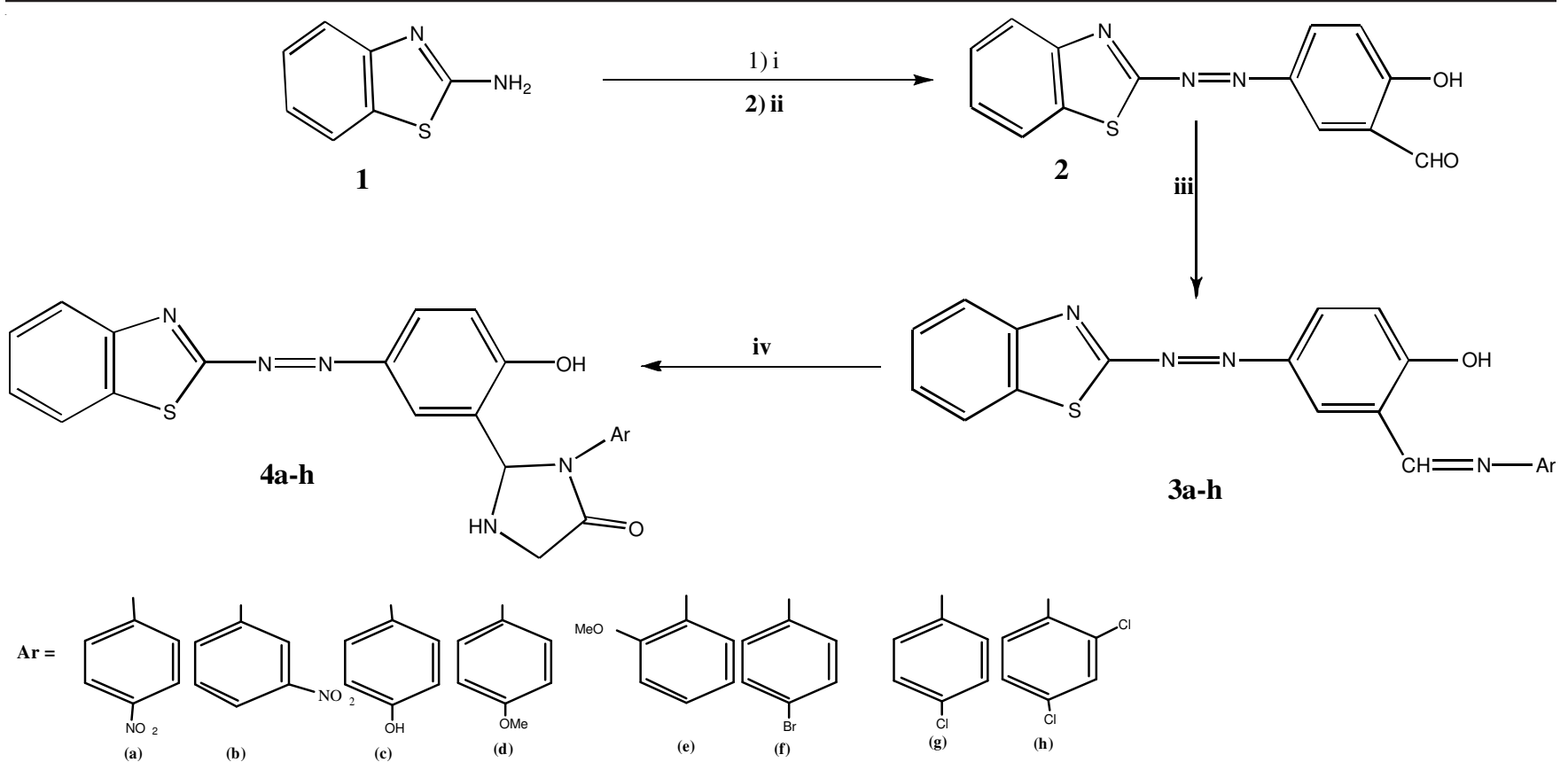

Scheme-I: Synthesis of imidazolidines, Reagents and conditions (i) $\mathrm{NaNO}_{2}, \mathrm{H}_{2} \mathrm{SO}_{4}, 0{ }^{\circ} \mathrm{C}$; (ii) 2-hydroxybenzaldehyde, $\mathrm{NaOH} 10 \%, 5{ }^{\circ} \mathrm{C}$; (iii) $\mathrm{Ar}-\mathrm{NH}_{2}, \mathrm{EtOH}, \mathrm{MW}(300-350 \mathrm{~W}), 25-30 \mathrm{~min}$; (iv) glycine, THF, MW (550-610 W), 20-25 min

\begin{tabular}{|c|c|c|c|c|c|c|}
\hline \multicolumn{7}{|c|}{$\begin{array}{c}\text { TABLE-1 } \\
\text { PHYSICAL PROPERTIESOFTHESYNTHESIZEDCOMPOUNDS }\end{array}$} \\
\hline Product & Colour & $\mathrm{R}_{\mathrm{f}}$ (developer) & m.p. $\left({ }^{\circ} \mathrm{C}\right)$ & Yield $(\%)$ & Time ( $\min )$ & MW (W) \\
\hline 2 & Dark brown solid & $0.68(n$-hexane/EtOAc, $1: 2)$ & $141-143$ & 60 & - & - \\
\hline $3 \mathbf{a}$ & Brown solid & $0.78(n$-hexane/EtOAc, $1: 2)$ & $221-223$ & 78 & 30 & 350 \\
\hline $3 b$ & Dark brown solid & $0.73(n$-hexane/EtOAc, $1: 2)$ & $184-186$ & 75 & 28 & 320 \\
\hline $3 c$ & Dark brown solid & $0.75(n$-hexane/EtOAc, $1: 2)$ & $176-178$ & 79 & 27 & 300 \\
\hline 3d & Brown solid & $0.88(n$-hexane/EtOAc, $1: 2)$ & $195-197$ & 73 & 26 & 300 \\
\hline $3 e$ & Dark brown solid & $0.70(n$-hexane/EtOAc, $1: 2)$ & $199-201$ & 77 & 30 & 310 \\
\hline $3 f$ & Dark brown solid & $0.83(n$-hexane/EtOAc, $1: 2)$ & $173-175$ & 69 & 30 & 320 \\
\hline $3 g$ & Brown solid & $0.75(n$-hexane/EtOAc, $1: 2)$ & $206-208$ & 76 & 28 & 320 \\
\hline $3 \mathbf{h}$ & Brown solid & $0.70(n$-hexane/EtOAc, $1: 2)$ & $163-165$ & 77 & 26 & 310 \\
\hline $4 a$ & Orange solid & 0.69 (n-hexane/EtOAc, 1:2) & 281(dec.) & 75 & 24 & 600 \\
\hline $4 b$ & Solid yellow & $0.71(n$-hexane/EtOAc, $1: 2)$ & $322-324$ & 78 & 23 & 600 \\
\hline $4 c$ & Dark orange solid & $0.71(n$-hexane/EtOAc, $1: 2)$ & $219-221$ & 75 & 23 & 550 \\
\hline 4d & Dark orange solid & 0.69 (n-hexane/EtOAc, 1:2) & $134-136$ & 78 & 22 & 550 \\
\hline $4 e$ & Yellow solid & $0.68(n$-hexane/EtOAc, $1: 2)$ & $198-200$ & 74 & 24 & 570 \\
\hline $4 f$ & Orange solid & $0.73(n$-hexane/EtOAc, $1: 2)$ & $228-230$ & 79 & 25 & 590 \\
\hline $4 g$ & Yellow solid & $0.74(n$-hexane/EtOAc, $1: 2)$ & $180-182$ & 78 & 25 & 600 \\
\hline $4 h$ & Dark brown solid & $0.72(n$-hexane/EtOAc, 1:2) & $233-235$ & 76 & 23 & 610 \\
\hline
\end{tabular}

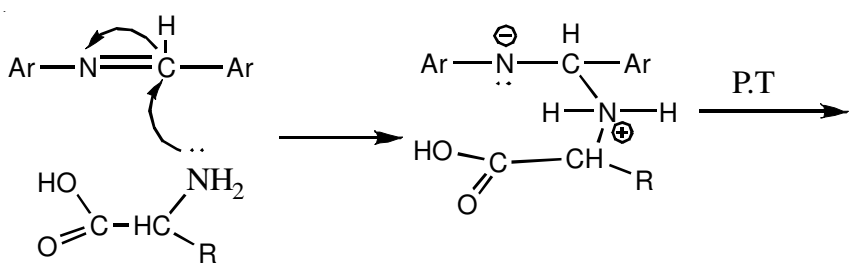

The structures of imidazolidine compounds $\mathbf{4 a - h}$ were proven by their ${ }^{1} \mathrm{H}$ NMR spectra $\left(500 \mathrm{MHz}, \mathrm{DMSO}-d_{6}\right)$ which showed the peak for the methylene protons $\left(\mathrm{CH}_{2}\right)$ of imidazolidine ring as a doublet at $\delta 3.69,3.69,3.69,3.69,3.69,3.69$, 3.69 and $3.68 \mathrm{ppm}$, respectively. The (N-CH-N) proton of imidazolidine ring appeared as a doublet at 6.63, 6.62, 6.63, $6.63,6.77,6.54,6.52$ and $6.74 \mathrm{ppm}$, respectively. The (Ar-H) protons at $\delta 6.67-8.14 \mathrm{ppm}$, the $(\mathrm{N}-\mathrm{H})$ proton of imidazolidine ring as a singlet at $8.14,8.12,8.12,8.15,8.15,8.15,8.15$ and $9.29 \mathrm{ppm}$, respectively. The $(\mathrm{O}-\mathrm{H})$ proton as a singlet at 9.48 , 9.56, 9.53, 9.52, 9.62, 9.64, 9.17 and 9.62 ppm, respectively. The methoxy protons $-\mathrm{OCH}_{3}$ in compounds $\mathbf{4 d}$ and $\mathbf{4 e}$ appeared as a singlet at $\delta 3.94$ and $3.83 \mathrm{ppm}$, respectively.

Moreover, the (CHNS) elemental analysis results were assigned to the $\delta(\mathrm{N}-\mathrm{H})$ bending of the imidazolidine ring. The benzothiazolic $v(C=N)$ str. appeared in the range 1616-1602 $\mathrm{cm}^{-1}$. within $\pm 0.4 \%$ of the theoretical values and in good agreement with the proposed chemical structures for compounds $\mathbf{4 a - h}$. 
Antibacterial activities: The antibacterial activities of the newly synthesized imidazolidines $(\mathbf{4 a}-\mathbf{h})$ were evaluated by the agar diffusion method [29] using representative standard strains of Gram (+) and Gram (-) bacteria on tryptic soya agar media (Table-2). Dimethyl sulfoxide was used as solvent for the test compounds.

\begin{tabular}{ccc}
\multicolumn{4}{c}{ TABLE-2 } \\
$\begin{array}{c}\text { ANTIBACTERIAL ACTIVITY OF COMPOUNDS (4a-h) } \\
\text { AND GENTAMYCINAS CONTROL DRUG }\end{array}$ \\
\hline \multirow{2}{*}{ Product } & $\begin{array}{c}\text { Staphylococcus aureus } \\
\text { (Gram-positive) }\end{array}$ & $\begin{array}{c}\text { Escherichia coli } \\
\text { (Gram-negative) }\end{array}$ \\
& 22 & 0 \\
$\mathbf{4 a}$ & 18 & 15 \\
$\mathbf{4 b}$ & 19 & 0 \\
$\mathbf{4 c}$ & 16 & 16 \\
$\mathbf{4 d}$ & 20 & 0 \\
$\mathbf{4 e}$ & 0 & 10 \\
$\mathbf{4 f}$ & 22 & 0 \\
$\mathbf{4 g}$ & 21 & 17 \\
$\mathbf{4 h}$ & 0 & 0 \\
DMSO & 15 & 15 \\
Gentamycin & & \\
\hline
\end{tabular}

Imidazolidine compounds $4 \mathbf{a}, 4 \mathbf{b}, \mathbf{4 c}, \mathbf{4 d}, \mathbf{4 e}, 4 \mathrm{~g}$ and $4 \mathrm{~h}$ were found to be greater activity than gentamycin against Gram-positive bacteria. Moreover, compounds $\mathbf{4 d}$ and $\mathbf{4 h}$ also showed better activity to the control drug against Gram-negative bacteria.

\section{Conclusion}

The microwave irradiation is efficient technique including short reaction time and high yield in comparison with the classical thermal method. The rates of reactions of imines with glycine for formation of imidazolidines are approximately equal. All synthesized imidazolidines could be converted to the corresponding phenol salts which are completely soluble in water. The synthesized imidazolidines appeared higher biological action against Gram-positive bacteria than that of Gram-negative bacteria. Imidazolidine compounds $\mathbf{4 a}, \mathbf{4 b}, \mathbf{4 c}$, 4d, $4 \mathbf{e}, 4 \mathrm{~g}$ and $\mathbf{4 h}$ were found to be greater activity than gentamycin against Gram-positive bacteria, while compounds 4d and $\mathbf{4 h}$ showed better activity to the control drug against Gramnegative bacteria.

\section{ACKNOWLEDGEMENTS}

The authors thank Faculty of Science, University of Tarbiat Modares, Iran for their significant assistance in ${ }^{1} \mathrm{H}$ NMR and elemental analyses.

\section{REFERENCES}

1. A. Husain, A. Ahmad, S.A. Khan, M. Asif, R. Bhutani and F.A. AlAbbasi, Saudi Pharm. J., 24, 104 (2016); https://doi.org/10.1016/j.jsps.2015.02.008.

2. H. Zhang, Q. Shen and L. Lu, Tetrahedron Lett., 52, 349 (2011); https://doi.org/10.1016/j.tetlet.2010.11.061.

3. Y. Brouillette, V. Lisowski, J. Guillon, S. Massip and J. Martinez, Tetrahedron, 63, 7538 (2007); https://doi.org/10.1016/i.tet.2007.05.057

4. A.S.H. Da Silva Guerra, D.J. Do Nascimento Malta, L.P.Morais Laranjeira, M.B. Souza Maia, N. Cavalcanti Colaço, M. Do Carmo Alves De Lima, S.L. Galdino, I. Da Rocha Pitta and T. Gonçalves-Silva, Int. Immunopharmacol., 11, 1816 (2011); https://doi.org/10.1016/j.intimp.2011.07.010.
5. A.L. Braga, F. Vargas, C.C. Silveira and L.H. De Andrade, Tetrahedron Lett., 43, 2335 (2002); https://doi.org/10.1016/S0040-4039(02)00300-3.

6. J.K.A.L. Neves, S.P.S. Botelho, C.M.L. de Melo, V.R.A. Pereira, M.C.A. de Lima, I. da Rocha Pitta, M.C.P. de Azevedo Albuquerque and S. Lins Galdino, Parasitol. Res., 107, 531 (2010); https://doi.org/10.1007/s00436-010-1886-y.

7. G.G. Muccioli, N. Fazio, G.K.E. Scriba, W. Poppitz, F. Cannata, J.H. Poupaert, J. Wouters and D.M. Lambert, J. Med. Chem., 49, 417 (2006); https://doi.org/10.1021/jm050977k.

8. K. Sztanke, S. Fidecka, E. Kedzierska, Z. Karczmarzyk, K. Pihlaja and D. Matosiuk, Eur. J. Med. Chem., 40, 127 (2005);

https://doi.org/10.1016/j.ejmech.2004.09.020.

9. T. Arai and Y. Ogino, Molecules, 17, 6170 (2012); https://doi.org/10.3390/molecules17056170.

10. L. Balewski, F. Saczewski, P.J. Bednarski, M. Gdaniec, E. Borys and A. Makowska, Molecules, 19, 17026 (2014); https://doi.org/10.3390/molecules191017026.

11. R.B. De Queiroz, F.L. De Carvalho, D.V. Da Fonsêca, J.M. BarbosaFilho, P.R.R. Salgado, L.L. Paulo, A.B.M. De Queiroz, L.C. De Morais Pordeus, S.A. De Souza, H.D. Da Silva Souza, B.F. Lira and P.F. De Athayde-Filho, Molecules, 20, 974 (2015); https://doi.org/10.3390/molecules20010974.

12. M.M. Ghorab, N.E. Amin, M.S.A. El Gaby, N.M.H. Taha, M.A. Shehab and I.M.I. Faker, Phosphorus Sulfur Silicon Rel. Elem., 183, 2918 (2008); https://doi.org/10.1080/10426500802505440.

13. J.K.A.L. Neves, M. de Lima, V.R.A. Pereira, C.M.L. de Melo, C.A. Peixoto, I.R. Pitta, M.C.P.A. Albuquerque and S.L. Galdino, Exp. Parasitol., 128, 82 (2011); https://doi.org/10.1016/j.exppara.2011.01.021.

14. C.G. Mortimer, G. Wells, J.-P. Crochard, E.L. Stone, T.D. Bradshaw, M.F.G. Stevens and A.D. Westwell, J. Med. Chem., 49, 179 (2006); https://doi.org/10.1021/jm050942k.

15. T. Wróbel, U. Kosikowska, A. Kaczor, S. Andrzejczuk, Z. Karczmarzyk, W. Wysocki, Z. Urbañczyk-Lipkowska, M. Morawiak and D. Matosiuk, Molecules, 20, 14761 (2015);

https://doi.org/10.3390/molecules200814761.

16. D. Mene and M. Kale, Curr. Org. Synth., 13, 41 (2015); https://doi.org/10.2174/1570179412999150723154128.

17. M. Al-Talib, Y.A. Al-Soud, M. Abussaud and S. Khshashneh, Arab. J. Chem., 9, S926 (2016); https://doi.org/10.1016/j.arabjc.2011.09.003.

18. B. Rajeeva, N. Srinivasulu and S.M. Shantakumar, E-J. Chem., 6, 775 (2009); https://doi.org/10.1155/2009/404596.

19. S. Saeed, N. Rashid, P.G. Jones, M. Ali and R. Hussain, Eur. J. Med. Chem., 45, 1323 (2010); https://doi.org/10.1016/j.ejmech.2009.12.016.

20. P. Vicini, A. Geronikaki, M. Incerti, B. Busonera, G. Poni, C.A. Cabras and P. La Colla, Bioorg. Med. Chem., 11, 4785 (2003); https://doi.org/10.1016/S0968-0896(03)00493-0.

21. L.C. Cabrera-Pérez, I.I. Padilla-Martínez, A. Cruz, J.E. Mendieta-Wejebe, F. Tamay-Cach and M.C. Rosales-Hernández, Arab. J. Chem., https://doi.org/10.1016/j.arabjc.2016.02.004.

22. A. Abarca, R. Ballesteros, M. Chadlaoui, J. Miralles, J.V. Murillo and D. Colonna, Tetrahedron, 57, 10111 (2001); https://doi.org/10.1016/S0040-4020(01)01053-5.

23. M. Charde, M. Shinde, A. Welankiwar and K. Jitendra, Int. J. Pharm.. Chem., 5, 104 (2015)

24. R.K. Gill, R.K. Rawal and J. Bariwal, Arch. Pharm., 348, 155 (2015); https://doi.org/10.1002/ardp.201400340.

25. M. Singh and S.K. Singh, Anticancer. Agents Med. Chem., 14, 127 (2014); https://doi.org/10.2174/18715206113139990312.

26. A. Darque, A. Dumètre, S. Hutter, G. Casano, M. Robin, C. Pannecouque and N. Azas, Bioorg. Med. Chem. Lett., 19, 5962 (2009); https://doi.org/10.1016/j.bmcl.2009.08.013.

27. D. Romani and S.A. Brandán, Comput. Theor. Chem., 1061, 89 (2015); https://doi.org/10.1016/j.comptc.2015.03.018.

28. Q.A. Acton, Azo Compounds: Advances in Research and Application, Scholarly Paper Edition, Atlanta (2011).

29. N.S. Egorove, Antibiotics Scientific Approach, Mir Publishers, Moscow (1985). 